\title{
Study on the Production Process of a Fruit and Vegetable Distribution Center
}

\author{
Xingxiang Tao, Juntao Li, Xiaohuan Xiang \\ School of Information, Beijing Wuzi University, Beijing, China \\ Email address: \\ 260486736@qq.com (Xingxiang Tao),862974337@qq.com (Juntao Li),313809457@qq.com (Xiaohuan Xiang)
}

\section{To cite this article:}

Xingxiang Tao, Juntao Li, Xiaohuan Xiang. Study on the Production Process of a Fruit and Vegetable Distribution Center. Science Research. Vol. 4, No. 2, 2016, pp. 48-54. doi: 10.11648/j.sr.20160402.15

Received: April 5, 2016; Accepted: April 14, 2016; Published: April 25, 2016

\begin{abstract}
Because of the rapid development of the Internet industry in China, many traditional industries have been overturned. Traditional buyers and sellers can transmit information over the network, eventually complete a transaction through the logistics process. "Fresh" is an important component of thousands of kinds of commodities. Fresh e-commerce is no longer part alien to the society. An e-commerce company in Shanghai, China, committed to the high earners in the city with fresh ingredients since 2005. But the existing modes of production can hardly meet the scale of production and production requirements, we need to explore suitable production process optimization methods to improve the production efficiency so as to meet the requirements of its scale of production. For the existing problems of the fruit and vegetable distribution center, the author of this article will use the methods based on lean management to optimize the production and logistics operations. For the production process mainly uses value stream analysis methods, to eliminate waste in the production process, to speed the flow of products, then to improve work efficiency. Optimize processes based on the analysis eventually.
\end{abstract}

Keywords: Fresh E-commerce, A Fruit and Vegetable Distribution Center, Value Stream Analysis Methods

\section{Introduction}

Fresh e-commerce is no longer alien to the society. The e-commerce experts have been circling round the topic of agricultural products for a quite long time. In 2011, China's vegetable output was 679 million tons, accounting for $60 \%$ of the world production, and the vegetable became China's staple agricultural product for the first time [1]. China's per capita vegetable has reached $370 \mathrm{~kg}$ per person per year, ranking first in the world [2]. Because of the particularity of fruit and vegetable products, production and processing region is an indispensable part of a fruit and vegetable distribution center. It's more a fruit and vegetable processing and distribution center than a fruit and vegetable distribution center. Fruit and vegetable distribution enterprise is strict with the processes in the distribution center. Fruit and vegetable should not be subjected for a long time, and consumers require fresh, high quality fruits and vegetables. Fruit and vegetable distribution center processes include the production process, the process design should consider the production processing and distribution centers combine basic functionality. The design of fruit and vegetable distribution center workflow whether reasonable has a great influence on the working efficiency. Fruit and vegetable should not be subjected for a long time, if the job is inefficient, it will lead to product quality issues, adverse impact on consumers and companies.

Lean management theory is the most comprehensive method of the research on production logistics system. With Toyota Manufacturing System (TPS), Toyota completed a qualitative leap in the manufacturing level. Lean management theory, derived from the Toyota production system, is very useful not only in the manufacturing industry, but also has a very important significance for non-manufacturing. The production process in the fruit and vegetable distribution center, and the processes associated with the production and processing of fruit and vegetable distribution center is also particularly important. Vigorously developing safe and efficient green supply chain of fresh agricultural products becomes an important factor in achieving sustainable development of the national economy. [3] In this paper, through lean management method of the value analysis on the production of fruit and vegetable distribution center logistics, and then optimize the processes based on the analysis. 
Thereby eliminating waste in the production process, speeding up product flow, improving production process efficiency. Provide consumers with the freshest fruits and vegetables more efficient.

\section{Value Stream Management}

Value Stream Management [4], which using Value Stream Mapping. And value stream mapping, which including information flow, material flow and processing parameters, is drawing the production activities before submitting products to customers. Then define the activities which created value. The activities are which can increase value-added by changing the form of the product, displaying the activities which do not create value, and then establish the appropriate team to improve the organization of non-value-added activities and optimize the value-added activities.

Identify the value stream which can start from the three tasks:

1. From conceptual design to production process, all the details of the design and engineering tasks to solve the problem.

2. From receiving the order to develop a detailed production schedule to deliver information management tasks throughout the process.

3. From raw materials to final products, send to users of the material transformation tasks.

The focus on this paper is that the entity made from raw material to end product production process [5]. In a fruit and vegetable distribution center [6], the production process requires raw materials from fruit storage areas, while provided accessories from accessories area. Under the cooperation of the two sectors, production and processing area would complete the whole production process. So the value stream analysis of this paper focuses on three processes:

1. The process of delivering the raw material from the storage areas to the production and processing areas.

2 . The process of the materials processing and delivering the materials from the material area to the production and processing areas.

3. The process of the processing in the production and processing areas.

All production activities are divided into three categories by value stream analysis:

1. The activities which can create value clearly.

2. The activities which can not create value, but which can't be omitted.

3. The activities, which can not create value, should be omitted immediately.

It can be clearly reflected the part which needs to be improved through the value stream diagram. The value stream diagram is a current state diagram which describing every process state, logistics, information stream and value stream between deferent processes by some simple symbols. Once we've identified areas which need to be improved, and then describe a future state diagram to show the improved directions and results of the value stream. Value Stream Analysis is the first to analyze the current situation of the operation. That is analyzing the "current state". Starting from the customer terminal, firstly, understanding the customer's needs and beats, and then studying the operation of the process in every process, from downstream dating back upstream until suppliers. Analyze value-added and non-value-added activities of each process, including the preparation, processing, stock, material transfer method and so on, and record the corresponding time, understand and analyze the path and method of of logistics information transmission, and distinguish and identify the causes of waste according to the analysis, to eliminate waste and provide continuous improvement goals. Finally, according to the actual situation, design a new value stream, indicate the direction of future operations.

\section{The Value Stream Analysis of Processing Areas}

Processing zone is a core operation of the distribution center in the district. In this area staff will process the raw material for simple production according to the schedule request. In a fruit and vegetable distribution center, the area of the processing zone about 30 meters long and 20 meters wide. Within this area, there are about six tables. Staff is divided into a group of six people around the table to operate. Sometimes the staff will be adjusted according to production requirements. There are two ways of processing in a distribution center: the large cargo and free patching. The large cargo is the commodity packaging specification which defined by a company, such as a box contains 10 pears. Free patching is commodity combinations according to the customers' willingness, such as a box contains 5 pears and 5 apples. After the sorting of large cargo is completed, then they can be sent directly to the delivery zone for delivery. The freedom patching should be sent to the commodity container for the second sorting, and then they can be sent to the delivery zone.

The problems in the processing zone are these:

1. The assignment of the work table is unreasonable. Each process takes long or short, the process with the long duration will become a bottleneck in the production.

2. Raw materials are scattered heap beside the table. These materials are either necessary or unnecessary for the processing. Way of randomly items placing and putting the unnecessary raw materials will reduce the production efficiency.

3. It will produce a large number of product backlog aside the table when processing has finished, and it will affect the production process.

We should do value analysis of the processing zone at first to solve these problems above. After knowing the using of each process, the number of staff and the inventories in process, we can identify the waste in the production process, and eliminating the waste is the goal of process optimization. 
The workflow of the workbench: a worker unpacks the requiring raw materials and auxiliary materials, and then 3 to 5 workers package the items according to the specification. Workers should check the vegetables and fruits for damage before packaging, singling out the damaged ones and packaging the undamaged. After packaging, they should be handed to the weighing staff to weigh, weighing staff will supply or take out according to the weight. Workers will label the packages if the weights are up to standard. Then a standard specification commodity is finished.

There may be a few differences in the workflow according to the production of commodities. For example, in the process of producing a standard specification of grapes, the workers should crop the large strings into small strings. And in the process of producing a standard specification of lychee, the lychees should be plucked from the branches one by one. Those fruits of slightly larger specification such as apples and pears don't need these processes, but they have to be bagged. Much larger specifications such as coconuts and watermelon may not have to be cut or bagged in the early work, but later they need more cushioning materials in packaging box.

Because many different kinds of fruits in the production and processing area, doing time statistics and value stream analysis for all these will spend a lot of time. The processing procedures of some types of fruits are alike, so we can divide the specifications of fruit into three categories: 1. The small size, such as raisins, grapes, lychees, etc. 2. Medium specification such as pears, apples, avocado, etc. 3 . The large size such as watermelon and coconut. After the classification, we can do processes value analysis of the fruits in accordance with the three specifications.

\subsection{The Processes Value Analysis of the Small Specification Fruits}

1. The processes value analysis of the small specification fruits, such as the grapes, the workflow is shown in the following figure:

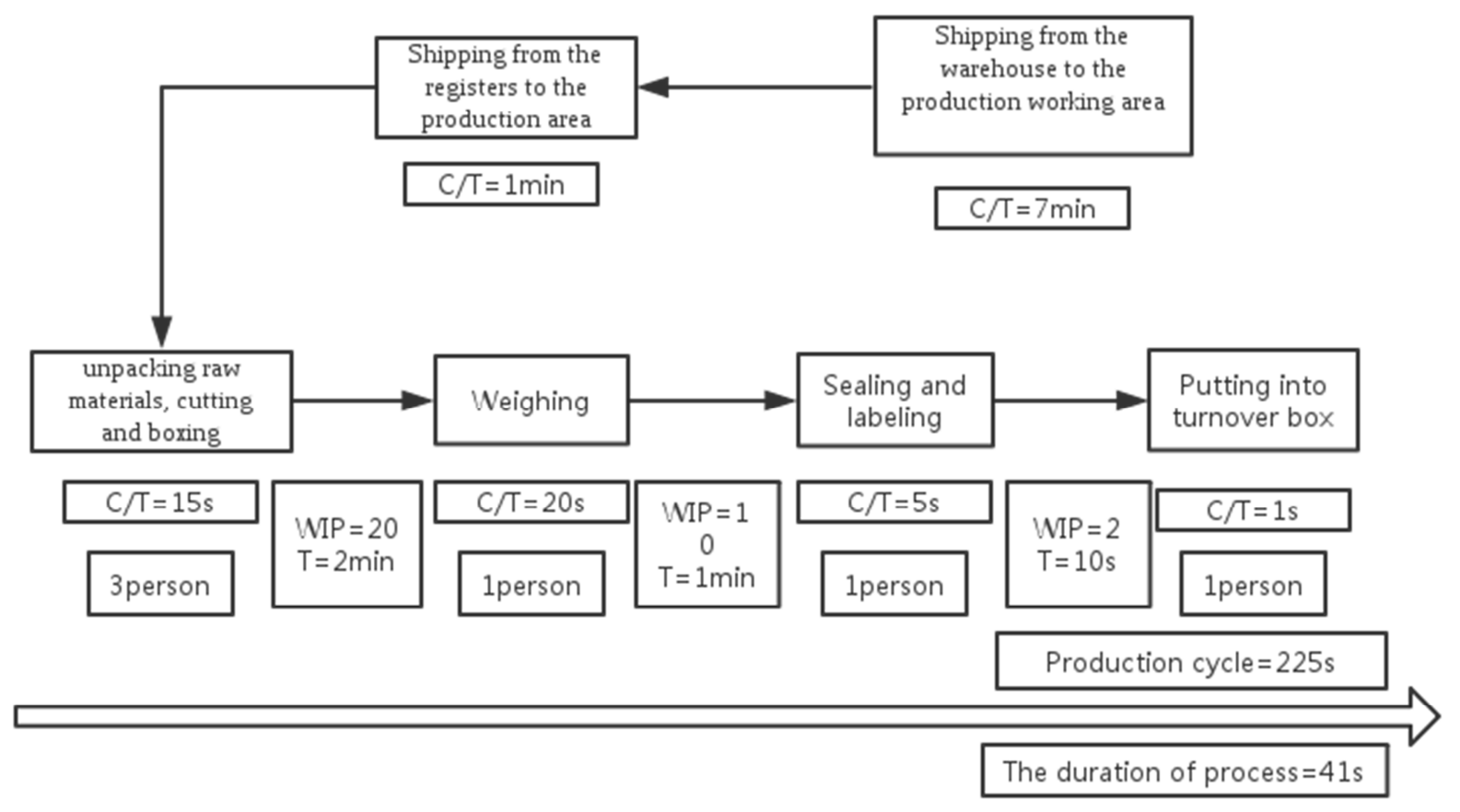

Figure 1. The workflow of the small specification fruits.

As shown in the Figure 1, the raw materials shipped from the warehouse to the production working area take 7 minutes, and from the scratch area to the production area takes 1 minute. In the production area, there are three workers unpacking the boxes of material. The big bunch of grapes should cut into small bunches before boxing, and pick defective fruits before boxing, packing a box takes 15 seconds for each worker. After boxing, the boxes will be delivered to the next worker to weigh them, the weighing workers weigh each box of grapes, if the box is overweight, the fruits will be decreased from the box, and if the weight is not enough, the worker need to make up the difference, the process will take 20 seconds. After weighing, the boxes will be transferred to the next worker to seal and label. This process will take 5 seconds. After labeling is completed, these fruits boxes will be put into the crates to wait to be shipped to the shipping staging area.

In this process, the first procedure is three workers to operate, while the second procedure only one person, which would lead to a large number of in-process inventory between the first procedure and the second procedure [7]. The subsequent procedure takes time for 20 seconds, the third procedure only takes 5 seconds, the system will also lead to product inventory between processes. The allocation of time is unreasonable, then the production flow will be blocked. The production of a box of standard specification product takes 225 seconds, but the actual value of the production process is only 41 seconds. 80 percent of the time 
was wasted. Balance is needed to increase productivity.

2. Adjusting the production processes of the small specification fruits

Production processes can be adjusted to that unpacking and boxing raw materials for a worker, two workers are responsible for weighing, and one worker are responsible for sealing, labeling and mounting crates. The workflow is shown in the following figure:

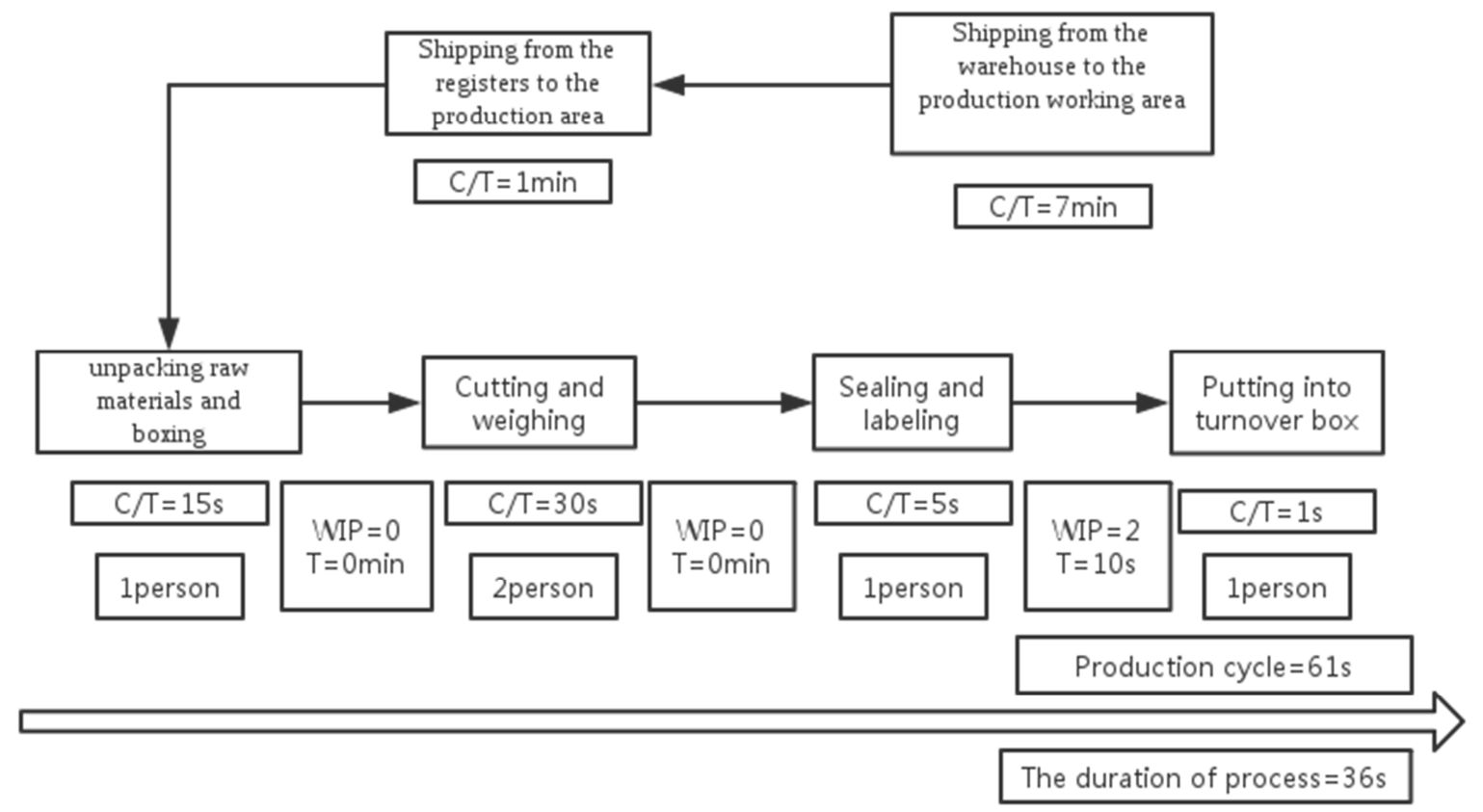

Figure 2. The improvement workflow of the small specification fruits.

As shown in the Figure 1, in the production area, there is a worker unpacking and boxing the boxes of material, the first procedure is cut down the cutting of operating procedures, so the first procedure time is 15 seconds. Two workers are assigned to weigh and crop the fruits in the second procedure, so one staff member will take 30 seconds. As the number of the workers in the first procedure is half of the number in the second procedure, it happened that the operational capability of the first procedure meets the job requirements of the next procedure. Then there would be no in-process inventory between the two processes. In the third procedure, the operating time is 5 seconds, every 30 seconds the subsequent procedure can produce two products which the third procedure requires, so the third procedure would deal with the two products for only 10 seconds. The operational capability of the third procedure is far greater than the second one. Therefore, the way of two simultaneous operations will be used in the third procedure, and the third procedure of the in-process inventory is only two products, the scratch time is 10 seconds. Due to the surplus capacity in the third procedure, the workers of the third procedure are assigned put the products into containers and finish operations.

Comparison on before and after optimization as shown in the Table 1:

Table 1. Comparison on before and after optimization of the workflow of the small specification fruits.

\begin{tabular}{llllll}
\hline & Production Cycle & Procedure Time & In-process Inventory & Staff Member & The Number of Products Per Minute \\
\hline Before & $225 \mathrm{~s}$ & $41 \mathrm{~s}$ & $20 / 10 / 2$ & 5 & 3 \\
After & $61 \mathrm{~s}$ & $36 \mathrm{~s}$ & 2 & 4 & 4 \\
Comparison & $27 \%$ & $87 \%$ & $18 \%$ & $80 \%$ & $130 \%$ \\
\hline
\end{tabular}

The improved production cycle is only $27 \%$ of the original one, and the in-process inventory is only $18 \%$ of the original one. When the speed of the product flow is increasing, the production efficiency will increase. Once the speed of the product flow is increasing, the problems on the production line emerge earlier, the earlier solution to the problem will be further high production efficiency.

The pre-processing workflow of lychee production is that pick off lychees from the branches. This process requires the manpower and time are relatively more. The next production processes are similar to the operation of the grapes. So only the number of staff in the first procedure should be adjusted, all the staff around the table could be adjusted to join the first procedure, after the first procedure, the staff of other procedures can go on their productions. The operation is similar with the grapes', so it can be processed in accordance with grapes production mode. And some other small specification fruits can be processed in accordance with this model.

\subsection{The Processes Value Analysis of the Medium Specification Fruits}

1. The processes value analysis of the medium specification fruits, such as the avocado. The workflow is shown in the following figure: 


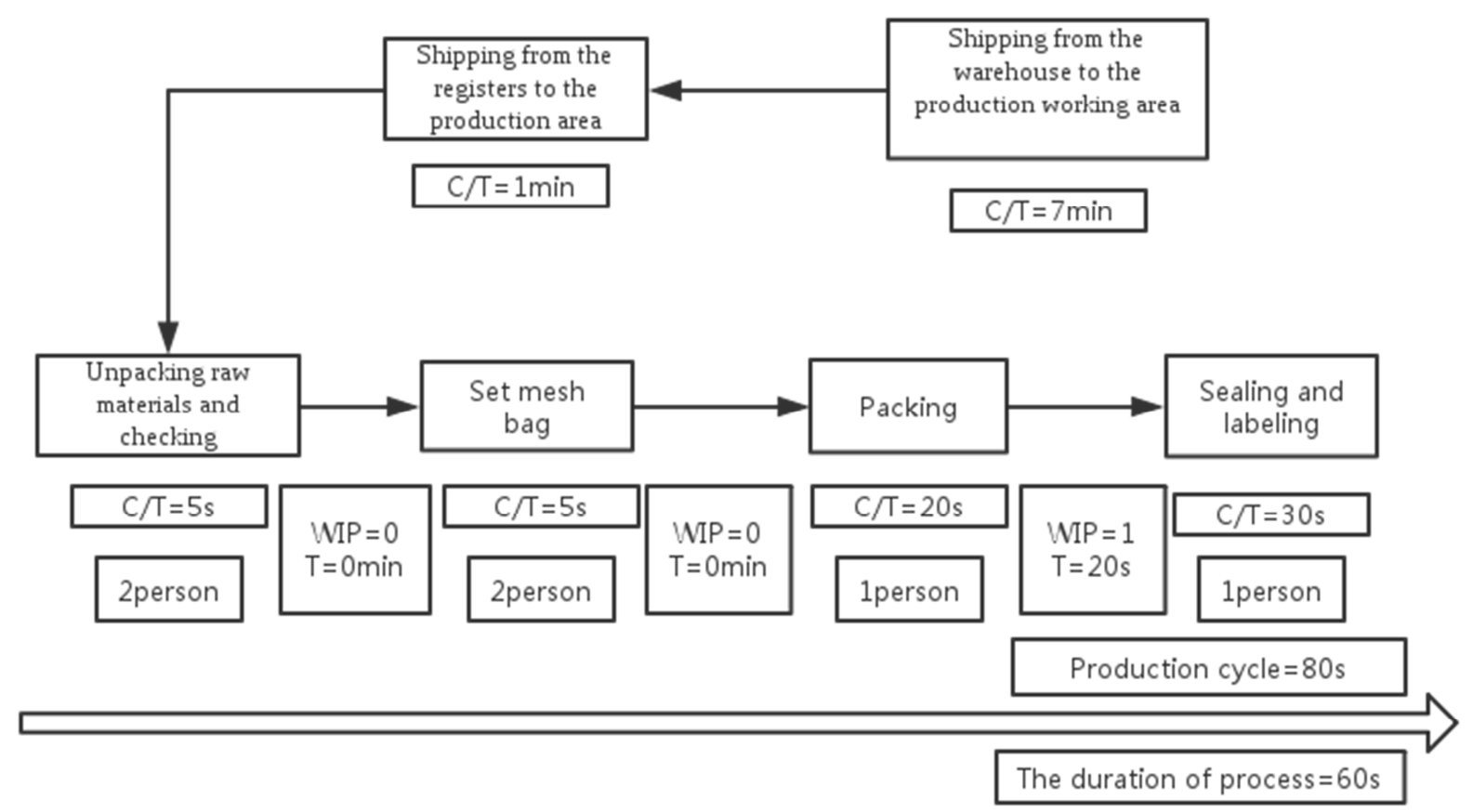

Figure 3. The workflow of the small specification fruits.

As shown in the Figure 3, when the raw materials are transported from the temporary storage area to the production area, they will be unpacked and checked. Each staff member needs 5 second to check an avocado, after this procedure the other staff will set mesh bags of the qualified avocado, the operation will take the staff 5 second, then the staff will put the avocadoes into the boxes, in accordance with the specifications of a box of 20 avocadoes, it will take 20 seconds, after this, the next staff will seal and label the boxes, these boxes will be put on the trays at last.
Because of unpacking, checking and setting mesh bag require less time than packing, sealing and labeling, so packing, sealing and labeling is the bottleneck of the entire production process. So we need to carry out the entire process of production balance adjustment, and speed up the production flow of the product.

2. Adjusting the production processes of the medium specification fruits

The adjusted workflow is shown in the following figure:

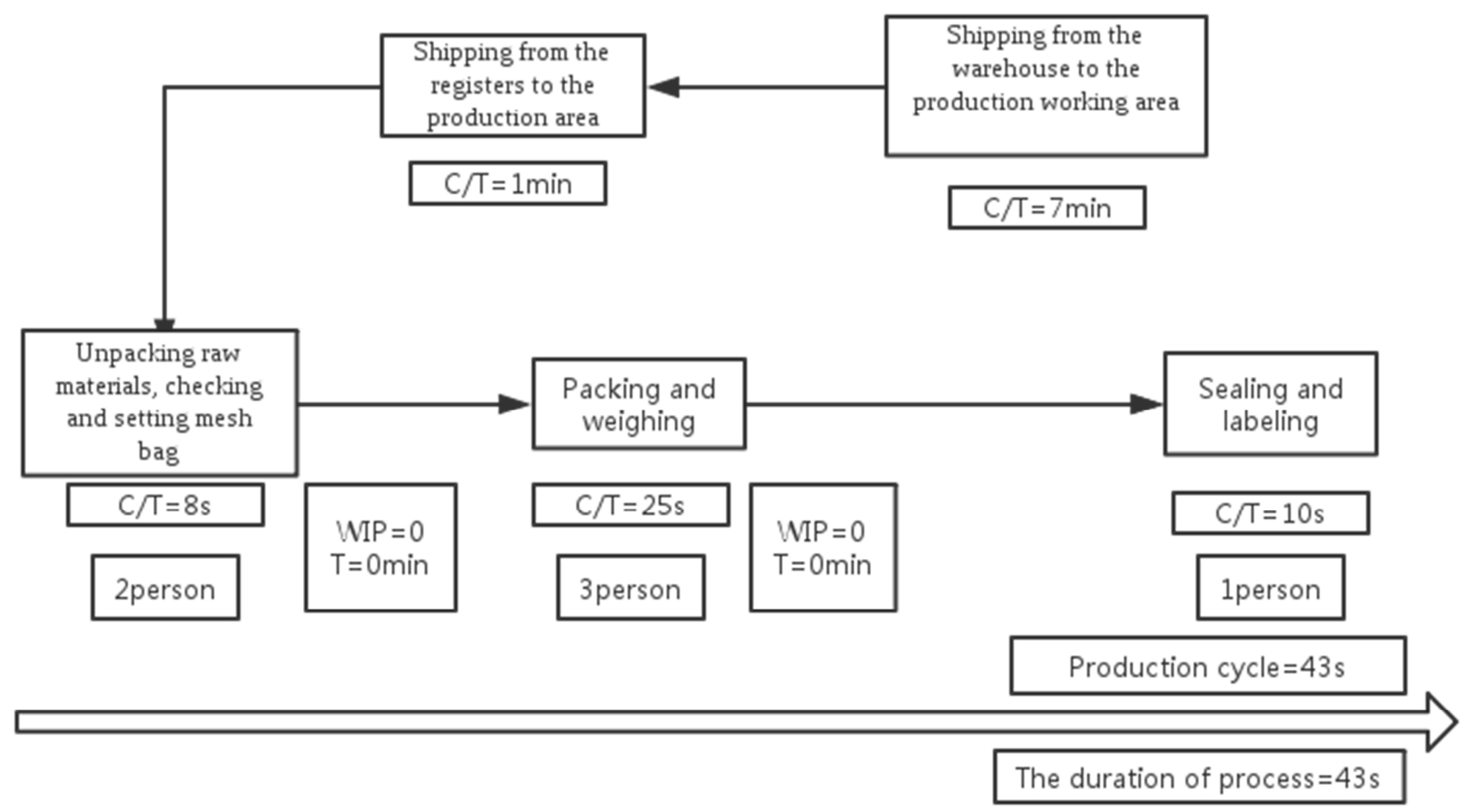

Figure 4. The improvement workflow of the medium specification fruits. 
As shown in the Figure 4, to combine the two processes of unpacking the raw material, checking and setting mesh bag, the workers will do the process of setting mesh bags as soon as they complete checking, thus the whole operation eliminates a process of transmission. The original of each movement takes 5 seconds. After the merger of the two actions now takes only 8 seconds. After the completion of bagging and later the products will be delivered to the packing and weighing staff, three workers will be adjusted to do the work of packing and weighing, because packing and weighing take workers much time. Every 24 seconds, the packing and weighing operation of three boxes of avocado could be completed. In-process inventory is zero, the staging time is zero. The time of sealing and labeling is 10 seconds, and 30 seconds could deal with three boxes of avocado. The capacity of the third procedure can meet the supply of the second procedure. Five workers are needed in the whole operation.

Comparison on before and after optimization as shown in the Table 2:

Table 2. Comparison on before and after optimization of the workflow of the medium specification fruits.

\begin{tabular}{lllllll}
\hline & Production Cycle & Procedure Time & In-process Inventory & Staff Member & The Number of Products Per Minute \\
\hline Before & $80 \mathrm{~s}$ & $60 \mathrm{~s}$ & 1 & 6 & 2 \\
After & $43 \mathrm{~s}$ & $43 \mathrm{~s}$ & 0 & 5 & 6 \\
Comparison & $53 \%$ & $71 \%$ & & $83 \%$ & $300 \%$ \\
\hline
\end{tabular}

As can be seen from the table, after optimization, the production targets of production cycle, the process time and the number of staff have been greatly improved. It is shown that the efficiency is promoted three times before optimization.

There's not much market for the large specification fruits during my research, and the production processes of the large specification fruits are simpler than the small and medium ones, there is no analysis of the large one here. The improvements in the production process for the small and medium specification fruits are only the first step in a long march of ten thousand li, because the process is impossible to achieve perfect proportions, there is much room for improvement. Adjusting according to the action is the core of lean management process optimization [8].

\section{Conclusion}

In this paper, through the in-depth research of a fruit and vegetable distribution center, and a thorough research on the production process is studied. Some wastes of the production process have been eliminated by using the value stream analysis of lean management theory. Balancing and merging the production processes to reduce the number of the in-process overstocks, so that products flowing become smoother, and the productivity has risen greatly.

Lean management is a mature theory [9], and it has been so widely used in manufacturing, but the application of the fruit and vegetable distribution center is not very extensive. An important principle of lean management is the pursuit of perfection, Taiichi Ohno [10] also said there would always have room for improvement. This paper optimized the production process of a fruit and vegetable production logistics distribution center for only one time, although the verification results show good results achieved than before, but there is still abundant room for improvement. Many times optimization improvements will achieve better results.

\section{Acknowledgements}

This paper is supported by the Funding Project for Technology Key Project of Municipal Education Commission of Beijing (ID: TSJHG201310037036); Funding Project for Beijing Intelligent Logistics System Collaborative Innovation Center; Funding Project for Beijing key laboratory of intelligent logistics system; Funding Project of Construction of Innovative Teams and Teacher Career Development for Universities and Colleges Under Beijing Municipality (ID: IDHT20130517), and Beijing Municipal Science and Technology Project (ID: Z131100005413004);Funding Project for Beijing philosophy and social science research base specially commissioned project planning (ID: 13JDJGD013).

\section{References}

[1] ZHANG P, ZHANG H, CHEN SH, et al. Status and considerations for development strategy of the industry of logistics and storage of fruits and vegetables in China [J]. Storage \& Process, 2013, 13(4): 1 -5.

[2] Vegetable production can't continue to expand blindly [J]. Farmer's Digest, 2013(2): 61.

[3] Yingtang LI, Zhong QIAO. Analysis of the Influencing Factors and Key Driving Force concerning the Efficiency of Green Supply Chain of Fruits and Vegetables [J]. Asian Agricultural Research, 2014, 07: 17-21.

[4] Andrea Bonaccorsi, Gionata Carmignani, Francesco Zammori. Service Value Stream Management (SVSM): Developing Lean Thinking in the Service Industry [J]. Journal of Service Science and Management, 2011, 44.

[5] Adnan Hj. Bakri, Abdul Rahman Abdul Rahim, Noordin Mohd. Yusof, Ramli Ahmad. Boosting Lean Production via TPM [J]. Procedia - Social and Behavioral Sciences, 2012.

[6] Wei Hu, Ye Hou, Longwei Tian, Yuan Li. Selection of logistics distribution center location for SDN enterprises [J]. Journal of Management Analytics, 2015, 23. 
[7] Ford Andria L, Williams Jennifer A, Spencer Mary, McCammon Craig, Khoury Naim, Sampson Tomoko R, Panagos Peter, Lee Jin-Moo. Reducing Door-to-Needle Times Using Toyota's Lean Manufacturing Principles and Value Stream Analysis. [J]. Stroke, 2012, 4312.

[8] Roberto Arbulu, Iris Tommelein, Kenneth Walsh, James Hershauer. Value stream analysis of a re-engineered construction supply chain [J]. Building Research \& Information, 2003, 312.
[9] AMALIA VENERA TODORUT, CECILIA IRINA RĂBONȚU, DORU CÎRNU. LEAN MANAGEMENT - THE WAY TO A PERFORMANT ENTERPRISE [J]. Annals of the University of Petrosani: Economics, 2010, X3.

[10] M. L. Emiliani, P. J. Seymour. Frank George Woollard: forgotten pioneer of flow production [J]. Journal of Management History, 2011, 171. 